\title{
Rapport sur la 3ème Conférence Internationale sur l'Epididyme.
}

\author{
Barry T. HINTON ${ }^{1}$, Joël R. DREVET ${ }^{2}$ \\ ${ }^{1}$ The University of Virginia, Health Science Center, Department of Cell Biology, Charlottesville, VA, USA. \\ 2 Université Blaise Pascal, CNRS UMR 6547- Génétique des Eucaryotes et Endocrinologie Moléculaire, Equipe \\ "Epididyme \& Maturation des Gamètes", Aubière, France.
}

La Conférence Internationale sur l'Fpididyme qui s'est tenue du 28 Mai 2002 au 1er Juin 2002 à Charlottesville (Virginie LSA) a été la 3eme édition d'une série de réunions se déroulant approximativement tous les 4 ans. Les précédentes manifestations étaient : Hong-Kong en 1992 et Newcastle (Australie) en 1998. L'objectif de ces réunions est de faire le point des recherches fondamentales conduites sur la physiologie de l'épididyme des mammifères et sur la maturation post-testiculaire des gamètes mâles. L'édition 2002 de la Conférence Internationale sur l'Epididyme a été organisée par les Dr. Barry T. Hinton et Terry T. Turner de l'Université de Virginie à Charlottesville (Virginie, USA). Le Comité Scientifique de cette réunion était composé de: Dr. Marie-Claire Orgebin-Crist (Vanderbilt University, Nashville, Tennessee, USA), Dr. Trevor G. Cooper (Université de Münster, Münster, Allemagne), Dr. Russel C. Jones (Université de Newcastle, Newcastle, Australie), Dr. Patrick Y.D. Wong (Chinese University de Hong Kong, Chine) et du Dr. Joël R. Drevet (CNRS/Université Blaise Pascal, Clermont2, Aubière, France). Afin de faciliter les interactions entre les congressistes le nombre de participants a été volontairement limité à 100. Le caractère international de la réunion a été confirmé par la grande diversité des nationalités représentées (Allemagne, Argentine, Australie, Brésil, Canada, Chine, Etats Unis, Finlande, lirance, Grande Bretagne, Inde, Israel, Sri Lanka, Thailande). De nombreux sponsors ont apporté leur soutien à cette manifestation (American Society of Andrology, University of Virginia School of Medicine, Lalor Foundation, Serono Foundation, Ernst Schering research Foundation, Marc Goldstein and Associates, Pharmacia \& Upjohn, Hamilton Thorne Research, Bio-rad, Fertility Solutions).

Huit symposia, deux conférences plénières et une session de posters (totalisant 45 posters laissés en place pour la durée du congrès) ont constitué le " core " central de la conférence. En parallèle, quatre groupes de discussion ont été organisés afin d'explorer les futures directions de recherche à privilégier.

Le symposium I concernait l'épithélium épididymaire. Le premier orateur, Dr. Bernard Robaire (McGill University, Montreal, Canada) a présenté des analyses de transcriptome réalisées sur les différentes régions épididymaires. Confirmant les résultats parcellaires acquis depuis de nombreuses années sur certains gènes modèles, cette approche globale du transcriptome épididymaire a montré qu'il existe de claires différences dans l'expression de plusieurs gènes en fonction des territoires épididymaires (tête, corps, queue de l'épididyme). II existe aussi de claires différences d'expression entre les régions épididymaires lorsque la stimulation androgénique est supprimée (castration). Suite à la perte des androgènes, beaucoup de gènes apoptotiques voient leur expression stimulée alors que l'expression de gènes pro-apoptotiques est au contraire diminuée. Le Dr. Danel Cyr (INRS-Institut Armand Frappier, Québec, Canada) a parlé d'adhésion cellulaire et d'intégrité des tubules épididymaires. Il a présenté des résultats concernant la régulation de l'expression de plusicurs protéines des jonctions gap dans l'épididyme. Les androgènes semblent réguler l'expression de la connexine 43 alors que les hormones thyroïdes semblent réguler le taux de transcription de son ARNm. En utilisant la technique de RT-PCR, Daniel Cyr a pu identifier les connexines $30.3,30.1$ et 32 dans l'épididyme de rat adulte et la connexine 26 dans l'épididyme de jeune rat. Beaucoup de ces connexines sont régulées au cours du développement. Sur la base de la présence de multiples connexines et de leur expression différentielle au cours de l'ontogenèse de 
l'épididyme du rat, Daniel Cyr a proposé qu'elles pourraient jouer des rôles différents dans le développement épididiymaire. Le Dr. Sylvie Breton (Massachusetts General Hospital, Charlestown, Maryland, USA) a parlé du trafic intracellulaire de l'hydrogène ATPase de type vacuolaire dans l'épithélium de l'épididyme. Elle a présenté des résultats concernant lc rôlc de cette enzyme dans la sécrétion de protons dans le canal défèrent de rat. Ce transporteur a été identifié au niveau des cellules claires de l'épithélium épididymaire. Il apparaît comme responsable de la maintenance du $\mathrm{pH}$ luminal acide de l'épididyme. Le trafic membranaire de ce transporteur dépend lui-même du pH luminal et de la modulation du cytosquelette d'actine cellulaire. Des études récentes ont montré que la protéine SNAP-23 était impliquée dans le ciblage du transporteur à la membrane apicale des cellules épididymaires. Il a été suggéré que des niveaux faibles de bicarbonate et un $\mathrm{pH}$ luminal acide joueraient un rôle dans la maintenance des spermatozoïdes dans un état quiescent durant leur passage dans l'épididyme.

Le symposium II concernait la régulation de l'expression des gènes dans l'épididyme. Les orateurs étaient Joël Drevet (CNRS/Université Blaise Pascal, Clermont-France, France), Gail Cornwall (Texas Tech University HSC, Lubbock, Texas, USA) et Barry T. Hinton (University of Virginia, Charlotesville , Virginia, US $\Lambda$ ). Les travaux présentés par J. Drevet concernaient la régulation de l'expression d'une famille de glutathion peroxydases (GPX) (enzymes clefs du métabolisme oxydatif et dans l'élimination des espèces oxygénées réactives issues du métabolisme aérobie) dans l'épididyme de souris. L'accent était porté su: l'étude de l'expression du gène GPX5 codant pour une GPX spécifique de l'épididyme. L'analyse du promoteur du gène GPX5 a révélé la présence de sitcs fonctionnels de réponse aux androgènes ainsi que de sites de fixation pour des facteurs de transcription des familles Ets (PEA3) et GATA. Chez la souris, le gène GPX5 donne naissance à au moins 2 messagers. A l'aide d'un anticorps anti-GPX5, 7 à 8 spots protéiques distincts sont retrouvés après électrophorèse bi-dimensionnclle des protéines épididymaires. Ces résultats suggèrent qu'il existe des patrons complexes d'expression et de maturation post-traductionnelle de GPX5. Des approches in vivo utilisant des modèles de souris transgéniques afin de décortiquer les éléments cis-activateurs du promoteur du gène GPX5 ont aussi été présentées. Un fragment de $5 \mathrm{~kb}$ des séquences flanquantes au gène GPX5 peut conduire l'expression d'un gène rapporteur dans la tête de l'épididyme. Ce promoteur peut donc être utilisé pour cibler l'expression d'une molécule donnée in vivo dans l'épididyme murin. (jail Cornwall a présenté des résultats concernant l'expression et le rôle des protéines épididymaires apparentées aux cystatines (CRES). Par le biais de souris transgéniques, le Dr. Cornwall a montré qu'un fragment de promotcur de $1.6 \mathrm{~kb}$ du gène CRES n'était pas suffisant pour permettre l'expression de CRES dans l'épididyme murin, mais au contraire contrôlait l'expression dans le testicule (dans les cellules germinales). Le Dr. Cornwall a aussi montré que CRES constituait une nouvelle classe d'inhibiteurs de protéases qui pourraient réguler l'activité d'enzymes qui interviennent dans le "processing" des pro-hormones. Barry Hinton a présenté des résultats concernant le rôle de facteurs testiculaires présent dans le fluide épididymaire (cf: facteur de croissance fibroblastique b-FGF) dans la régulation de l'expression des gènes dans le segment initial de la tête de l'épididyme. Cette régulation passerait par la voie de transduction faisant appel aux MAP kinases. Dans un autre domaine, le Dr. Hinton a aussi présenté des résultats concernant l'expression du transportcur de la L-carnithine (OCTN2). Il semblerait que l'expression de OCTN2 soit régulée par des stress osmotiques via la voie des MAP kinases et de facteurs de transcription de réponse à la pression osmotique.

Le symposium III était sous l'égide de la fondation Lalor. Les participants étaient soit des étudiants en thèse de Doctorat d'Université soit des chercheurs en séjour post-doctoral. Le premier intervenant était $\mathrm{M}^{\mathrm{me}}$ Petra Sipila (Faculty of Medicine, Turku, Finlande). Elle a présenté des résultats concernant la production et la caractérisation de cellules épithéliales d'épididyme de souris en cultures immortalisées. Ces cultures de cellules ont été dérivées de lignées de souris transgéniques exprimant sous le contrôle du promoteur gpx5 un oncogène transformant (Antigene-T du virus simien SV40). La majorité des cellules dérivées des épithéliums épididymaires en hyperplasie expriment des gènes spécifiques de l'épididyme murin. Ces cellules seront des outi]s très intéressants pour la caractérisation des gènes épididymaires. Le second intervenant était $\mathrm{M}^{\text {elle }}$ Caroline Osterhoff (IHF, Institute for Hormonc and Fertility Research, Université de Hambourg, Hambourg, Allemagne). Cette dernic̀re a présenté des travaux concernant l'analyse de la protéine épididymaire humaine HE6, une protéine trans-membranaire longue de 1014 résidus acides aminés ayant les caractéristiques d'une protéine à 7 domaines trans-membranaires. La plus forte homologie a été trouvée avec la superfamile des récepteurs couplés à la protéine G (Calcitonin/secretine/VIP). Le ligand de HE6 et son rôle exact dans l'épididyme restent à découvrir. Daniela Bomgardner (The University of Virginia, Charlottesville. USA) a parlé du rôle des gènes homéotiques dans la différenciation de l'épididyme au cours du développement embryonnaire et chez. l'adulte. Les gènes Hox chez la souris sont connus pour leurs rôles dans le développement des structures segmentées. Le Dr. Bomgardner a montré que l'épididyme de souris adulte exprime aussi de nombreux gènes Hox et certains de leurs co-facteurs. Ceci suggère 
que les gènes homéotiques pourraient être importants pour le maintien de la segmentation épididymaire.

Le symposium IV était axé sur les protéines épididymaires et leurs fonctions. Les orateurs étaient Jean-Louis Dacheux (INRA, Tours Nouzilly, France), Patricia Cuasnicu (Buenos Aire, Argentine) et Robert Sullivan (CHUL, Québec, Canada). Le Dr. Dacheux a évoqué le recours à la protéomique pour caractériser l'ensemble des protéines sécrétées par l'épididyme d'espèces animales d'intérêt agronomiques (étalon et taureau). L'étape de caractérisation passée, le réel challenge sera de comprendre le rôle de ces différentes protéines et leurs interactions. Le Dr. Cuasnicu a montré que la protéine épididymaire de rat, CRISP1 (aussi appelée protéine DE), était impliquée dans les événements de reconnaissance de l'ovocyte. Des acides aminés particuliers situés dans la protéine DE semblent jouer un rôle central dans cette fixation du spermatozoïde à l'ovocyte. La compréhension de la structure fine de la protéine $\mathrm{DE}$ et de son implication dans l'interaction spermatozoïde/ovocyte pourrait à terme être utilisée pour le développement de stratégies contraceptives. Le Dr. Sullivan a évoqué les caractéristiques d'une famille de protéines épididymaires qui sont transférées à la surface des spermatozoïdes durant la maturation épididymaire. La protéine p26h (chez l'homme) semble avoir une activité carbonylréductase, elle est fixée à la membrane plasmique du spermatozoïde via une ancre GPI. Des résultats expérimentaux suggèrent que la protéine, dépourvue de signal peptidique de sécrétion, est sécrétée dans la lumière du canal épididymaire via des "épididymosomes" (structures similaires aux prostasomes) qui sont des vésicules de sécrétion apocrines de l'épithélium épididymaire (et prostatique).

Le symposium $\mathrm{V}$ concernait la protection et le stockage des spermatozoïdes. Le Dr. Russel Jones (University of Newcastle, Australie) a évoqué l'épididyme aviaire et comparé sa fonction avec l'épididyme des mammifères. Il a montré que les canaux efférents de la caille japonaise réabsorbent le fluide testiculaire environ 8 fois plus rapidement que la même structure chez le rat. La fonction de l'épididyme chez la caille versus le rat est différente, mais dans les deux cas entièrement sous la dépendance des androgènes. Une protéine sécrétée dans l'épididyme semble jouer un rôle prépondérant dans la maturation des spermatozoïdes de caille. Le Dr. Roy Jones (The Babraham Institute, Cambridge, UK) a parlé des variations de lipides membranaires et de l'importance des phénomènes de diffusions moléculaires sur la membrane des spermatozoïdes au cours de leur maturation épididymaire. Ces changements entraînent des réarrangements subtils de la membrane plasmique des spermatozoïdes qui peuvent déterminer les aptitudes à féconder des gamètes mâles. La migration de protéines par simple diffusion entre les différents domaines de la membrane plasmique des spermatozoïdes ne suffit pas à expliquer l'étendu des modifications observées. Des phénomènes de " trapping " et la participation du cytosquelette cortical du spermatozoïde sont à envisager. Le Dr. John Aitken (University of Newcastle, Australie) a parlé de stress oxydant et de régulation de l'état redox dans l'épididyme. Les spermatozoïdes sont particulièrement sensibles aux attaques radicalaires à cause de la composition particulière de leur membrane riche en acides gras polyinsaturés lesquels sont la cible de choix des espèces oxygénées réactives. Les spermatozoïdes doivent donc être protégés de façon efficace contre les effets délétères des radicaux libres. Le Dr. Aitken a montré que les espèces oxygénées réactives d'une part peuvent être très dommageable aux spermatozoïdes, mais que d'autre part, elles semblent aussi jouer un rôle physiologique important durant la capacitation. L'activité redox ne semble pas restreinte aux seuls spermatozoïdes puisque des résultats expérimentaux suggèrent que le segment initial de la tête de l'épididyme serait aussi redox actif.

Le symposium VI concernait l'épididyme humain. Aux présentations scientifiques fondamentales ont été ici associées à des présentations plus cliniques. Le Dr. Marc Goldstein (Cornell University Medical College, New York, USA) a parlé du rôle de l'épididyme et des thérapies micro-chirurgicales en fertilité humaine. Il a décrit des méthodes de vaso-épididymostomie utilisant des techniques de microchirurgie chez l'homme et aussi sur des modèles animaux. Différentes méthodes ont été comparées quant à leur efficacité. Une version modifiée de la technique d'intussusception à deux sutures avec ouverture longitudinale donne des taux de paternité proche de 93\%. Le Dr. Alan Diekman (University of Arkansas for Medical Sciences, Little Rock AR, USA) a parlé des antigènes de surface des spermatozoïdes humains acquis au cours du transit épididymaire. Il a plus particulièrement décrit la caractérisation de l'antigène épididymaire humain SAGA1/CD52 et son éventuelle utilisation dans des stratégies contraceptives. La protéine SAGA-1 présente des modifications post-traductionnelles complexes (glycosylations) et est ancrée à la surface des spermatozoïdes par une liaison Glycosyl Phosphatidyl Inositol (ancre GPI). Elle semble être impliquée dans la reconnaissance et l'accrochage spermatozoïde/ovocyte. Le Dr. Christiane Kirchhoff (IHF, Institute for Hormone and Fertility, Université de Hambourg, Hambourg, Allemagne) a parlé de régulation de l'expression des gènes dans l'épididyme humain. Elle a présenté les caractéristiques d'expression de plusieurs gènes épididymaires humains identifiés à partir d'un criblage différentiel d'une librairie d'expression d'épididyme humain. En particulier, elle a décrit le gène HE1 qui code pour une protéine de liaison du cholesterol et le gène HE2 qui code, quant à lui, pour un peptide à activité anti-bactérienne spécifique de l'épididyme. Plusieurs protéines épi- 
didymaires présentant des homologies avec des protéines anti-bactériennes ont été récemment découvertes dans l'épididyme des Mammifères.

Le symposium VII était dévolu à l'exploitation de l'épididyme en tant que source ou cible potentielle de stratégies contraceptives. L.es orateurs étaient le Dr. Ursula Habenicht (Ernst Schering Research Foundation, Berlin, Allemagne), le Dr. Trevor Cooper (Université de Munster, Munster, Allemagne) et le Dr. Gary Klinefelter (US EPA, Research Triangle Park, North Carolina, USA). Le Dr. Habenicht a présenté les perspectives de l'industrie pharmaceutique dans le domaine de la contraception. Le Dr. Habenicht a souligné la nécessité de développer un contraceptif mâle et esquissé les approches prises par l'industrie dans ce domaine. Brièvement, les approches sont concentrées sur les aspects post-testiculaires de la maturation des spermatozoïdes. L'épididyme est donc au centre des investigations. Dans un premier temps, l'accent est mis sur les collaborations avec les organismes publics de recherches et les instituts de laçon à faire progresser l'étendue des connaissances sur cet organe de même que sur d'autres organes du tractus génital mâle (canaux efférents, canal défèrent, vésicules séminales). Un premier exemple de partenariat public/privé est en cours sur l'épididyme des mammifères (Réseau AMPPA, Ernst Schering Research Foundation and The Rockefeller Foundation). Le Dr. Cooper a présenté le phénotype épididymaire du modèle murin de $\mathrm{KO}$ c-ros (un récepteur à activité tyrosine kinase présentant des similitudes avec le récepteur de l'EGF). Les souris nulles pour c-ros présentent une absence de développement du segment initial de l'épididyme qui se traduit par une infertilité mâle à cause de défauts de morphologie et de motilité des spermatozoïdes. Le Dr. Cooper a présenté des résultats qui supportent l'idée que les défauts de motilité et de morphologie des spermatozoïdes des souris $c$-ros ${ }^{-1-}$ sont en partie dus au fait que les spermatozoïdes sont inaptes à réguler leur volume. Le glutamate pourrait jouer un rôle clef dans ces phénomènes de régulation du volume des spermatozoïdes. Le Dr. Gary Klinefelter a présenté des résultats concernant la protéine épididymaire SP22 qui est sensible à des composés toxiques. Il a montré que des anticorps dirigés contre SP22 pouvait inhiber la fécondation.

Le symposium VIII était consacré aux nouvelles directions de recherche sur l'épididyme. Le Dr. Miles Wilkinson (MD Anderson Cancer Center, Houston, Texas, USA) a évoqué la caractérisation in vivo de séquences régulatrices du gène à homéodomaine Pem. Ces séquences contrôleraient au cours du développement l'expression de gènes androgéno-dépendants dans les cellules de Sertoli et dans l'épididyme. Par transgenèse additive, le Dr. Wilkinson a montré que seulement $0,3 \mathrm{~kb}$ du promoteur proximal du gène Pem était suffisant pour diriger l'expression de Pem dans le testicule et dans l'épididyme. Avec ce promoteur minimum, l'expression d'un gène rapporteur a été trouvée restreinte à certaines régions de l'épididyme et aux cellules de Sertoli dans le testicule. Ce promoteur était aussi suffisant pour conférer une régulation hormonale et temporelle correcte. Ce promoteur pourrait donc être utilisé pour diriger l'expression d'un trasngène donné dans le testicule et dans l'épididyme. Le Dr. llpo Huhtaniemi (Université de Turku, Faculté de Médecine, Turku, Finlande) a présenté des lignées de souris transgéniques qui pourraient être utilisés comme modèles pour comprendre le développement et la fonction de l'épididyme. Deux lignées transgéniques ont été générées en exprimant dans l'épididyme de souris l'antigène grand T de SV40 sous le contrôle d'un fragment de $5 \mathrm{~kb}$ du promoteur du gène GPX5. Une lignée transgénique a été utilisée pour dériver des cellules épithéliales épididymaires immortalisées (voir le symposium $\mathrm{N}^{\circ} \mathrm{III}$ ). La seconde lignée transgénique présente une hyperplasie du segment initial de l'épididyme et les mâles sont infertiles. L'infertilité est due à un défaut de morphologie (angulation du flagelle et présence de structure en boucle) et de motilité des spermatozoïdes ressemblant étrangement au phénotype des souris KO c-ros. Cette lignée de souris transgéniques constitue un bon modèle de dysfonctionnement épididymaire. Le Dr. Daniel Jonhston (WyethAmherst Research, Women's Health Research Institute, Radnor, PA, USA) a évoqué les stratégies de développement de contraceptif mâle basée sur l'exploitation des banques génomiques humaines. Afin d'identifier de nouvelles cibles pour le développement de contraceptifs mâles, le Dr. Johnston a dressé un inventaire des techniques d'études qui pourraient être employées pour cribler les informations disponibles sur les bases de données publiques et privées (EST, transcription profiling, genomics and proteomics,...). La combinaison de ces différentes informations pourrait permettre d'identifier des cibles potentielles pour le développement de nouveaux contraceptifs.

La première conférence plénière, sous l'égide de la Société Américaine d'Andrologie (ASA) a été donnée par le Dr. Marie-Claire Orgebin Crist (Vanderbilt University, Nashville, Tennessee, USA). Cette dernière a présenté ses réflexions concernant les recherches sur l'épididyme à l'ère de la post-génomique. L'accent a été porté sur l'importante somme d'information maintenant disponible pour les scientifiques et sur la nécessité d'avoir recours à des approches intégratives. Le Dr. Orgebin-Crist a ensuite présenté les fruits de ses propres recherches concernant unc famille multiprotéique de lipocalines et leurs rôles dans la physiologie de l'épididyme et la maturation des spermatozoïdes. Lcs lipocalines sont de petites protéines extracellulaires qui ont pour ligands des rétinoïdes, des stéroïdes et des acides gras. La seconde conférence plénière était sous 
le patronage de la Société Internationale d'Andrologie (ISA). Elle a été donnée par le Dr. Patrick Wong (The Chinese University of Hong Kong, Hong Kong, Chine). Le Dr. Wong a présenté ses résultats concernant les aspects moléculaires, cellulaires et physiologiques des transports de fluides et d'électrolytes dans l'épididyme. Plus particuliérement, le Dr. Wong a présenté des études récentes sur les interactions existantes entre le transporteur d'anions CFTR (cystic fibrosis) et l'échangeur d'ion sodium/hydrogène de type 3 en ce qui concerne les mouvements de fluides au travers de l'épithélium épididymaire. Il a en outre montré que CFTR interagit aussi avec la protéine aquaporine 9, une protéine connue pour être impliquée dans les mouvements d'eau et localisée à la membrane apicale des cellules de l'épithélium épididymaire. Le Dr. Wong a suggéré que ces protéines pourraient être utilisées pour transporter des agents pharmacologiques (par exemple de potentiels contraceptifs) dans l'épididyme.

Une caractéristique unique de cette $3^{\text {eme }}$ édition de la réunion épididyme a été la session intitulée "exploration topique ". Quatre groupes de discussion chacun sous la houlette d'un animateur se sont réunis autour des thèmes suivants : 1/ De quelles molécules et de quels outils avons nous besoin pour approfondir notre compréhension du développement et de la fonction de l'épididyme ? (animateur : Dr. Kenneth Roberts, University of Minnesota, Minneapolis, USA) ; 2 / quels sont les aspects des fonctions épididymaires qui nécessitent plus d'avancées, donc plus d'investigations? (animateur : Dr. Terry Turner, University of Virginia, Charlotesville, USA) ; 3 / quels sont les écueils à l'utilisation de l'épididyme comme une cible pour le développement d'outils contraceptifs ? (animateur : Dr. Rex Hess, University of Illinois, Urbana, IL, USA) ; 4/ quels sont les aspects uniques à l'épididyme qui pourraient constituer des cibles potentielles pour le développement de contraceptifs mâles ? (animateur : Dr. Michael Holland, Monash Institute of Reproduction \& Devclopment, Melbourne, Australia). Chaque " leader " d'un groupe de discussion a ensuite présenté un bref rapport à la fin de la réunion des points majeurs sortis de ces séances de "brain storming ".

Enfin un résumé de cette zeme édition de la Conférence sur l'Epididyme et une actualisation de la progression des recherches florissantes sur cet organe accessoire du tractus génital mâle a été brossé par le Dr. David Hamilton (University of Minnesota, Chapel Hill, North Carolina, USA). Il est anticipé que la prochaine édition de la Conférence Internationale sur l'Epididyme ait lieu en Europe en 2006. 